\title{
Inverse correlation of kidney interstitial cells expansion with hemoglobin level and erythropoietin expression in single and repeated kidney ischemic/reperfusion injury in mice
}

\author{
Dian Prasetyo Wibisono ${ }^{1}$, Nur Arfian ${ }^{1}$, Muhammad Mansyur Romi ${ }^{1}$, Wiwit Ananda Wahyu Setyaningsih ${ }^{1}$, and Dwi \\ Cahyani Ratna Sari ${ }^{1,{ }^{*}}$ \\ ${ }^{1}$ Department of Anatomy, Faculty of Medicine, Public Health and Nursing, Universitas Gadjah Mada, Jalan Farmako, Sekip Utara, Sleman, \\ Yogyakarta 55281, Indonesia \\ *Corresponding author: dwi.cahyani@ugm.ac.id
}

SUBMITTED 6 March 2019 REVISED 4 April 2019 ACCEPTED 29 May 2019

\begin{abstract}
Ischemic/reperfusion injury (IRI) causes acute kidney injury that may lead to chronic kidney disease. We investigated the correlation between kidney interstitial cells expansion, hemoglobin level, and erythropoietin expression as the chronic effects of single and repeated kidney IRI in mice. We created an IRI model using male Swiss mice by clamping the bilateral renal pedicles. Subjects were divided into four groups that contained six mice each: control/sham operation, single acute IRI, single chronic IRI, and repeated IRI. Our results showed that the single chronic and repeated IRI groups significantly increased the tubular injury score, decreased the hemoglobin level, and increased erythropoietin expression compared with the control. Lower hemoglobin levels in all of the groups compared with the control was associated with erythropoietin resistance. In single chronic and repeated kidney IRI, there were decreased creatinine levels compared with the control. The decreased creatinine levels from the single acute IRI group to the single chronic IRI group, suggesting a repair phase of IRI starting on day 7 occurred in the single chronic IRI group. A macrophage marker, CD68, and an inflammatory mediator marker, MCP-1, significantly increased in all IR groups, indicating inflammation occurred due to IRI. In conclusion, chronic and repeated kidney IRI induced interstitial cells expansion and inflammation associated with anemia.
\end{abstract}

KEYWORDS anemia; chronic kidney disease; erythropoietin; fibrosis; ischemic/reperfusion injury

\section{Introduction}

Chronic Kidney Disease (CKD) is a major health problem in both developed and developing countries (Prodjosudjadi and Suhardjono 2009). The progression of this disease into end-stage renal disease is difficult to avoid and the mortality rate still remains higher than $20 \%$ per year, even with the availability of dialysis. Based on data published by the National Kidney Foundation, the number of patients treated with dialysis and kidney transplantation has increased dramatically in the United States. More than 400,000 Americans suffered from end-stage renal disease, with more than 300,000 needing dialysis (Go et al. 2004).

CKD is known as one of the possible complications of Acute Kidney Injury (AKI). Kidney ischemic/reperfusion injury (IRI) can cause AKI and more than $70 \%$ of AKI cases will develop into CKD (Bonventre and Yang 2011). Reactive oxygen species can cause kidney insufficiency (Arfian et al. 2012), by inducing interstitial cells expansion and extracellular matrix production, which inhibits tubular epithelial cell proliferation in kidney IRI models (Kim et al. 2010). Interstitial area expansion leads to kidney interstitial fibrosis, which is known as the main charac- teristic of progressive kidney disease (Strutz and Zeisberg 2006).

Kidney fibrosis is mediated by fibroblast accumulation and dysfunction. Fibroblast dysfunction causes anemia due to the reduction of erythropoietin (EPO), the hormone that stimulates erythropoiesis (Asada et al. 2011). One study using in situ hybridization and transgenic mice found that EPO was produced by interstitial fibroblast at the inner cortex and outer medulla (Obara et al. 2008). Patients with CKD also show increases of inflammatory molecules; for example, C-reactive protein (CRP), and groups of cytokines such as IL1, IL6 and TNF alpha (Bergström and Lindholm 2000; Stenvinkel 2001). Proinflammatory cytokines can cause EPO resistance by disrupting iron metabolism (Donovan et al. 2005).

Subtotal nephrectomy and unilateral ureteral obstruction are known as models for CKD, whereas IRI is known as a model for AKI in mice (Arfian et al. 2012; Asada et al. 2011). In this study, the IRI model was used to induce CKD, especially in the chronic period of IRI. IRI could also induce CKD and kidney fibrosis after 12 days based on the histopathological findings, as shown by fibroblast 
proliferation as well as myofibroblast expansion, which is also associated with anemia (Asada et al. 2011). Studies on the chronic effect of single and repeated kidney IRI and the process of how AKI develops into CKD in this model are still rare. Therefore, it is necessary to investigate the chronic effect of kidney IRI, especially interstitial cells expansion (fibroblasts and pericytes). The correlation of interstitial cells' expansion with hemoglobin concentration and EPO expression in this model is also rarely investigated, so it is equally important to be studied.

\section{Materials and methods}

This was a quasi-experimental study with a post-test only controlled group design using 24 Swiss male mice, three months old, weighing 30-40 grams. Mice were obtained from the Integrated Research and Analytical Laboratory (Laboratorium Penelitian dan Pengujian Terpadu), Universitas Gadjah Mada, Yogyakarta, Indonesia. The animals were randomized and maintained under standard laboratory conditions and given access ad libitum to an animal diet and tap water. All experimental procedures were conducted in accordance with the Medical and Health Research Ethics Committee, Faculty of Medicine, Universitas Gadjah Mada, with expediency number KE/FK/845/EC.

\subsection{Kidney ischemic/reperfusion injury model}

Animal subjects were divided into four groups that contained six mice each: control/sham operation (SO), ischemic/reperfusion 1 (IR1), ischemic/reperfusion 12 (IR12), and ischemic/reperfusion 7-12 (IR7-12). Sham operation was used as the control, IR1 was used as the AKI model, IR12 was used as the chronic single IRI model, and IR7-12 was used as the repeated IRI model.

The kidney IRI model was performed under general anesthesia. Intraperitoneal injection of pentobarbital solution 1:10 (0.1mg/10 gramBW) was used as the anesthesia agent. In this model, we performed clamping of the right and left renal pedicles using a non-traumatic vascular clamp (Karl Hammacher GmbH, Solingen, Germany) for $30 \mathrm{~min}$. After $30 \mathrm{~min}$, the clamp was released and followed by reperfusion. The incised skin and peritoneum were then closed using surgical thread silk 3/0 (OneMedHealthcare, Surabaya, Indonesia).

In the SO group, no pedicle clamping was performed, only an abdominal incision followed by the closing of the incised skin. This group was euthanized on day 12. In the IR1 and IR12 groups, bilateral renal pedicle clamping was performed on day 0 and subjects were euthanized on days 1 and 12, respectively. In group IR7-12, bilateral renal pedicle clamping was performed on days 0 and 7 , followed by euthanasia on day 12 .

\subsection{Blood serum analysis}

Whole blood was obtained from the retro-orbital vein for the measurement of hemoglobin and creatinine levels. Whole blood samples for hemoglobin measurement were transferred into an EDTA tube, whereas whole blood samples for creatinine measurement were transferred into a 1.5 $\mathrm{mL}$ tube followed by centrifugation at $10,000 \mathrm{rpm}$ for 10 min. Supernatants were obtained as the blood serum and all samples were brought to the Clinical Pathology Laboratory, Faculty of Medicine, Public health, and Nursing, Universitas Gadjah Mada, for hemoglobin and creatinine measurement.

\subsection{Microscopic analysis}

\subsubsection{Tubular injury score}

To measure the tubular injury score, the kidneys were embedded in paraffin blocks and $4 \mu \mathrm{m}$ sections were stained with periodic acid-Schiff (PAS), examined with an Olympus CX22 light microscope (Olympus Corporation, Tokyo, Japan), and portrayed using the Optilab software at $100 \mathrm{x}$ magnification at the corticomedullary junction area. Tubular injury scores were determined using a semi-quantitative scoring system. Fifteen fields per kidney were examined and injuries were graded from 0-4 (0, normal; 1 , injury affecting $<25 \%$; 2 , injury affecting 25-50\%; 3, injury affecting 50-75\%; 4, injury affecting $>75 \%$ ), according to these variables: renal tubular atrophy and dilatation, intraluminal cast formation (reddish color inside the lumen in PAS staining), loss of brush border, accumulation of inflammatory cells, and intraluminal cast (Park et al. 2012).

\subsubsection{Immunohistochemical staining}

The kidneys were embedded in paraffin blocks and $4 \mu \mathrm{m}$ sections were made, deparaffinized and rehydrated using $100 \%$, $90 \%$, $80 \%$, and $70 \%$ alcohol, followed with the heating process in citrate buffer $(\mathrm{pH} 6)$ for antigen retrieval and blocking endogenous peroxidase using $\mathrm{H}_{2} \mathrm{O}_{2} 3 \%$ in PBS solution. The slides were then incubated using Background Sniper, rabbit $1^{\text {st }}$ monoclonal antibody PDGFR $\beta$ with 1:200 dilution (Abcam, ab32570, Cambridge, United Kingdom), TrekAvidin-HRP, $2^{\text {nd }}$ antibody anti-rabbit Trekkie Universal Link (Biocare Medical, STUHRP700, California, United States), and diaminobenzidine tetrahydrochloride (Biocare Medical, STUHRP700H L10). The results were analyzed using the ImageJ software, examined with a light microscope (Olympus CX22), and portrayed with the Optilab software at 400x magnification.

\subsection{Reverse transcriptase $P C R$ analysis}

\subsubsection{RNA extraction and CDNA synthesis}

Total RNA was extracted using Genezol (Geneaid GZR100, Geneaid Biotech Ltd, New Taipei City, Taiwan), followed by quantification of RNA concentration using spectrophotometry. We used 3,000 ng RNA for making cDNA. The cDNA was made using Rever Tra Ace ${ }^{\circledR}$ (Toyobo Cat. No. TRT-101, Osaka, Japan) and random primer (Toyobo Cat. No. 3801), with PCR conditions: $30^{\circ} \mathrm{C}$ for $10 \mathrm{~min}$ (denaturation), $42^{\circ} \mathrm{C}$ for $60 \mathrm{~min}$ (annealing) and $99^{\circ} \mathrm{C}$ for 5 min (extension). 


\subsubsection{Reverse transcriptase $P C R$ and electrophoresis}

Reverse transcriptase PCR was carried out to amplify the following specific cDNAs: erythropoietin (F: AGGAATTGATGTCGCCTCCA and R: AGCTTGCAGAAAGTATCCACTGTG); CD68 (F: CATCAGAGCCCGAGTACAGTCTACC and R: AATTCTGCGCCATGAATGTCC); monocyte chemoattractant protein1/MCP-1 (F: GGCATCACAGTCCGAGTCACAC and R: CTACAGACAACCACCTCAAGCACTTCTGT), and GAPDH (F: GGCACAGTCAAGGCTGAGAATG and R: TCTCGCTCCTGGAAGATGGTGA).

The reverse transcriptase PCR was performed by mixing $2 \mu \mathrm{L}$ cDNA, $12.5 \mu \mathrm{L}$ of Taq Master Mix (Bioron, Germany, Cat. No. S101705), $0.6 \mu \mathrm{L}$ of forward and reverse primer, and $9.3 \mu \mathrm{L}$ of PCR water. The cDNA were amplified to the following conditions: $94^{\circ} \mathrm{C}$ for $2 \mathrm{~s}$ (initial denaturation), $94^{\circ} \mathrm{C}$ for $10 \mathrm{~s}$ (denaturation), $60^{\circ} \mathrm{C}$ for 20 seconds (annealing), $72^{\circ} \mathrm{C}$ for $1 \mathrm{~min}$ (extension), and $72^{\circ} \mathrm{C}$ for $10 \mathrm{~min}$ (last extension) for 35 cycles. The PCR products were analyzed in $2 \%$ agarose gel along with a $100 \mathrm{bp}$ DNA ladder (Bioron Cat. No. 306009, Germany). Ex-

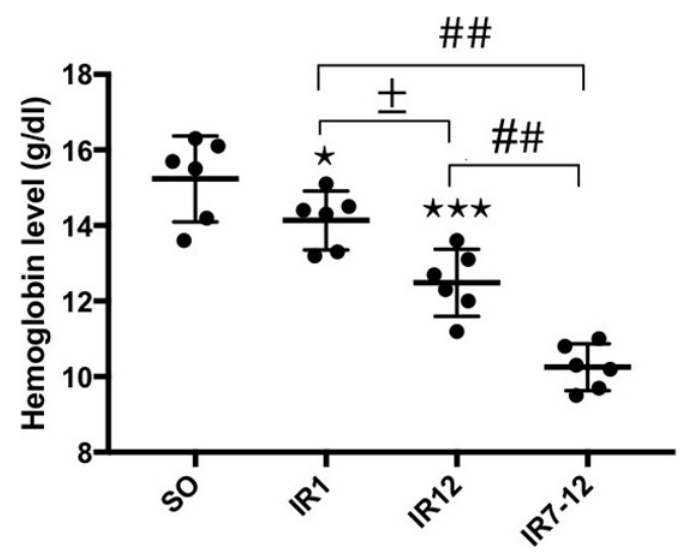

(a)

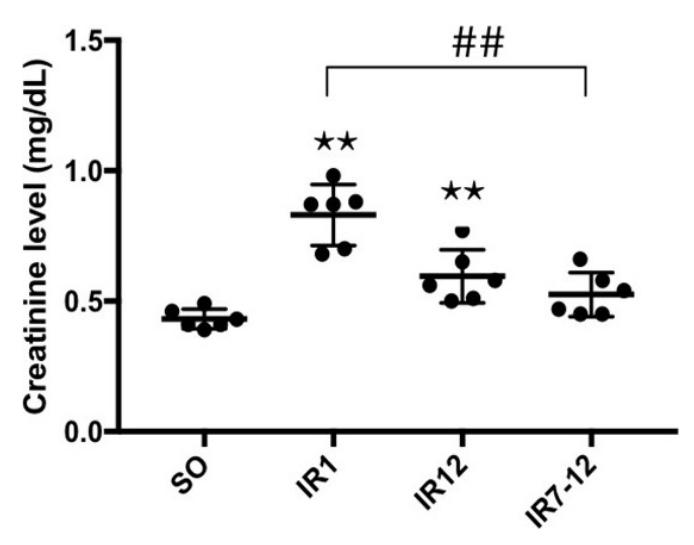

(b)

FIGURE 1 Results for mean hemoglobin levels (a) and mean creatinine levels (b). SO, control/sham operation; IR1, ischemic/reperfusion; IR12, ischemic/reperfusion 12; IR7-12, ischemic/reperfusion 7-12. Data were analyzed using one-way ANOVA $(p=0.000)$ and Kruskal Wallis $(p=0.000)$ tests. Asterisks show significance between SO and IRI groups $\left({ }^{* * *}, p<0.001 ;{ }^{* *}, p<\right.$ $\left.0.01 ;^{*}, p<0.05\right)$. Elbow connectors show significances between IRI groups (\#\#, $p<0.001 ; \pm, p<0.01 ; \#, p<0.05$ ). pressions of the gene were quantified with densitometry analysis using the ImageJ software. GAPDH was used as the housekeeping gene.

\subsection{Results analysis}

The data obtained were analyzed using the Shapiro Wilk test for distribution analysis. The Pearson correlation and Spearman correlation tests were used if the data were normally and abnormally distributed, respectively, with $p<$ 0.05 used to determine the level of significance. Multiple comparisons among the groups were made using one-way ANOVA and followed by post hoc LSD tests if the data were normally distributed. If the data were abnormally distributed, Kruskal Wallis and post-hoc Mann Whitney tests were used, with $p<0.05$ used to determine the level of significance.

\section{Results}

\subsection{Blood serum analysis}

Single chronic and repeated kidney IRI induced a decrease in hemoglobin levels, as shown in the significantly lower values in groups IR12 and IR7-12 compared with the SO group $(p<0.01)$. Single acute kidney IRI, represented by the IR1 group, also induced significantly lower hemoglobin levels compared with SO, but in smaller decreases compared with the IR12 and IR7-12 groups (Figure 1a). This result suggests that more chronic and more severe conditions caused greater decreases in hemoglobin levels. The decrease in hemoglobin levels in all groups compared with SO was associated with EPO resistance.

Compared with the SO group, single acute and single chronic kidney IRI induced significantly higher creatinine levels, as shown in both the IR1 and IR12 groups. However, repeated kidney IRI (IR7-12 group) did not show a significant difference compared with SO. Furthermore, single chronic (IR12 group) and repeated (IR7-12) kidney IRI showed lower creatinine levels compared with single acute (IR1 group) kidney IRI (Figure 1b). The decrease in creatinine levels from group IR1 to IR12 suggests a repair phase of IRI starting in day 7 occurred in the IR12 group.

\subsection{Microscopy analysis}

Kidney IRI induced tubular injury, which was characterized by intraluminal cast formation, dilatation, epithelial effacement, and brush border loss (Figure 2a). Higher magnification of PAS staining revealed that repairing of tubular epithelial cells occurred in the IR12 group, as indicated by brush border formation. However, the regeneration and tubular repair did not occur in repeated IRI (IR7-12 group). Quantification of the tubular injury score showed a significantly higher tubular injury score in the IR1, IR12, and IR7-12 groups compared with the SO group $(p<0.001)$. The IR1 and IR7-12 groups demonstrated higher tubular injury compared with the IR12 group (Figures 2a, 2b, and 2d). This condition suggested that the repair phase occurred in group IR12. Microscopically, the IR12 group revealed a regeneration process of tubular epithelial cells (Figures 2a, 2b, and 2c). 

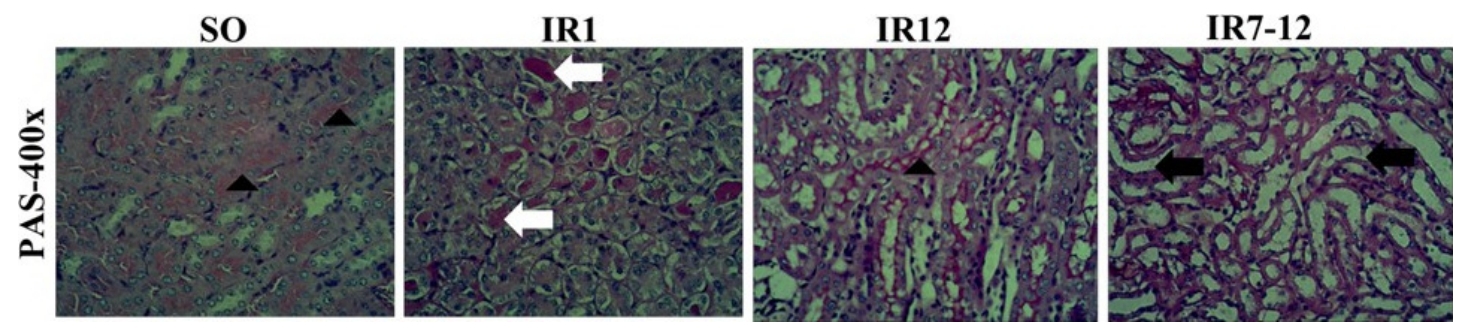

(a)
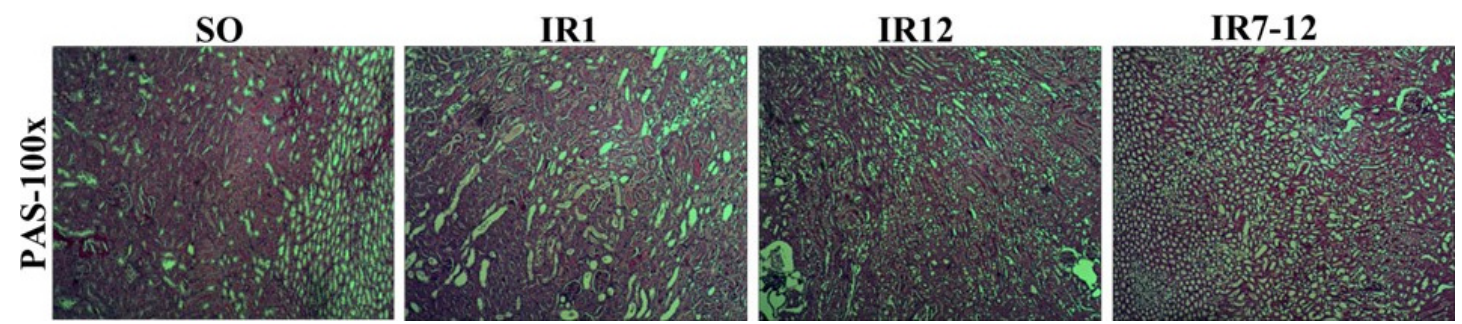

(b)
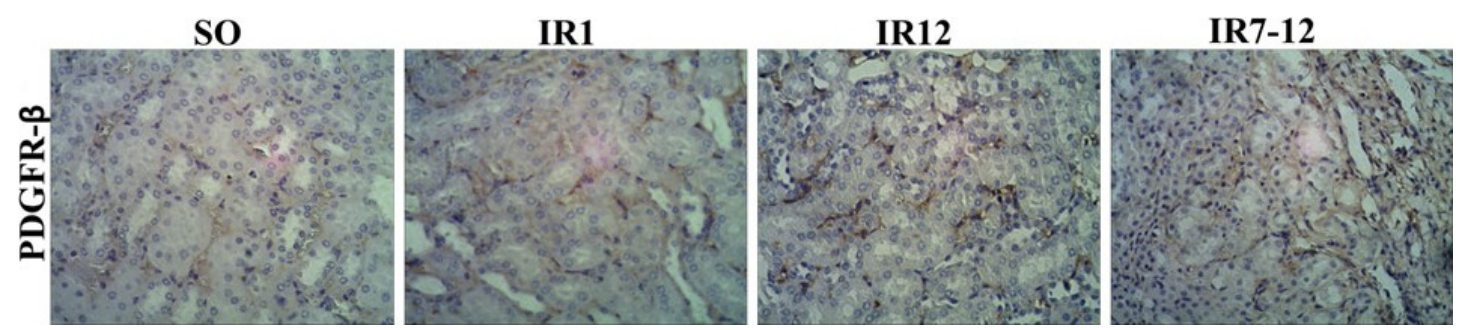

(c)

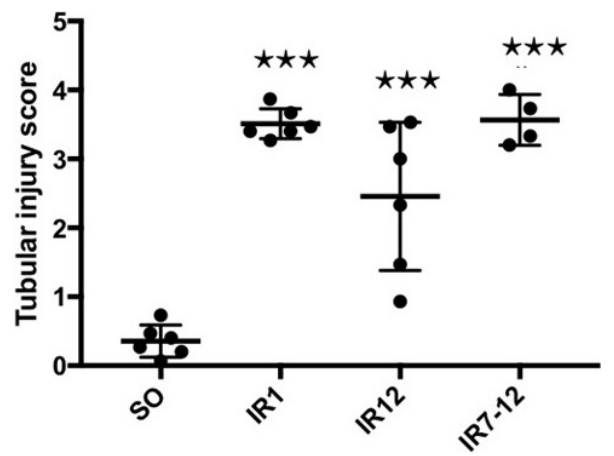

(d)

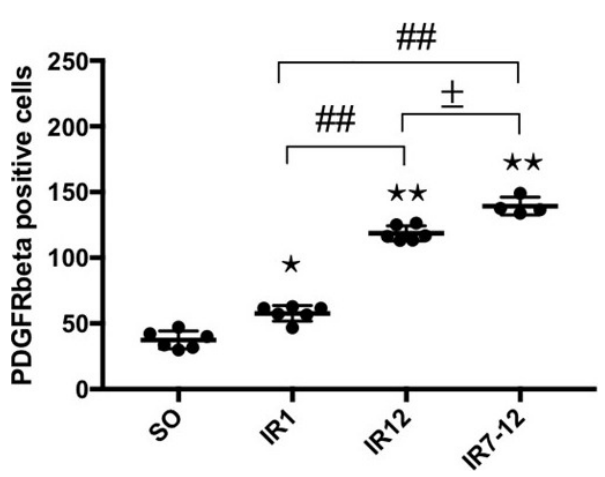

(e)

FIGURE 2 Histological quantification of tubular injury and PDGFR $\beta$-positive cells. (a) Representative figures of PAS staining. Control/sham operation (SO) group represents normal tubules with brush border (black triangles); tubular injury showed damage of tubular with cast formation (red color), brush border loss (white arrow) and epithelial cell effacement (black arrow) in ischemic/reperfusion (IRI) 1 (IR1), IR12, and IR7-12 groups. Brush border regeneration could be found in IR12 group. Scale bar $=100 \mu \mathrm{m}$. (b) Microscopic figures of kidney tissue. Scale bar $=100 \mu \mathrm{m}$. (c) Microscopic figures of PDGFR $\beta$ positive cells. PDGFR $\beta$-positive cells were shown by black arrow. Scale bar $=100 \mu \mathrm{m}$. (d) Results of tubular injury score. (e) Number of PDGFR $\beta$-positive cells. Tubular injury scores were analyzed using Kruskal Wallis test ( $p=0.001)$. Number of PDGFR $\beta$-positive cells were analyzed using one-way ANOVA $(p=0.000)$. Asterisks show significance between SO and IRI groups $\left(^{* * *}, p<0.001 ; * *, p<0.01 ;{ }^{*}, p<0.05\right)$. Elbow connectors show significances between IRI groups $(\# \#, p<0.001 ; \pm, p<0.01)$.

Kidney IRI induced a significant increase in the the number of positive PDGFR $\beta$ (fibroblast) cells in the IR1, IR12, and IR7-12 groups compared with the SO group. This condition was associated with a severe injury that leads to interstitial cell (fibroblasts and pericytes) expansion (Figures 2c and 2e).

\subsection{RT-PCR analysis}

Kidney IRI induced a significantly higher mRNA expression of EPO in the IR1, IR12, and IR7-12 groups com- pared with the SO group. This suggests that hypoxia, either in an acute or chronic condition, induces production of HIF and subsequently leads to EPO production (Figures 3a, 3b). RT-PCR of CD68 as a macrophage marker and MCP-1 as an inflammatory mediator demonstrated inflammation with macrophage infiltration occurred in the IR1, IR12, and IR7-12 groups. A significantly higher expression of CD68 mRNA was found in the IR1, IR12, and IR7-12 groups compared with the SO group (Figures 3a, $3 b$, and 3c), as well as MCP-1 (Figures 3a and 3b). Re- 
peated IRI, as represented by the IR7-12 group, showed a significantly higher MCP-1 mRNA expression compared with the IR1 and IR12 groups. These results suggest that chronic inflammation might be associated with EPO resistance in all groups, thus inducing anemia.

\subsection{Correlation test}

The Pearson correlation test between the number of PDGFR $\beta$-positive cells and hemoglobin level showed a significant, strong, negative correlation $(p=0.000 ; r=$ -0.822 ), whereas the Pearson correlation test between the number of PDGFR $\beta$-positive cells and EPO expression showed a significant, strong, positive correlation ( $p$ $=0.000 ; r=+0.795)$.

\section{Discussion}

This study found that a reduction of hemoglobin levels occurred in kidney IRI, which might be associated with inflammation and macrophage infiltration. Kidney IRI causes an imbalance between vasoconstriction and vasodilation mediators, which contributes to microvascular and tubular injury. Microvascular injury is represented by an increase of vasoconstriction and decrease of vasodilation response, endothelial and vessel smooth muscle cells injury, leucocyte-endothelial interaction, and inflammation. Tubular injury induces cytoskeleton damage, loss of polarity, apoptosis, and necrosis (Bonventre and Weinberg 2003).
The tubular injury might occur in an acute and chronic time after the kidney IRI model. Morphological changes in kidney microstructure demonstrate tubular injury characterized by a loss of the brush border at proximal tubules, intraluminal cast accumulation, tubular renal dilatation and atrophy, and accumulation of inflammatory cells in all IRI groups. Tubular injury also induces degradation of cellular debris into the tubular lumen and contributes to cast formation (Bayati et al. 1990), tubular lumen obstruction, and an increase in tubular pressure (Mason et al. 1977).

Tubular injury score quantification showed that the IR1, IR12, and IR7-12 groups had significantly higher scores than the SO group. The highest tubular injury score was in group IR7-12, followed by IR1. Mechanisms and processes that occurred in the IR1 group showed that tubular epithelial cell injury is the main mechanism in the initiation phase of kidney injury (Sutton et al. 2002). However, based on our result, in day 12 following IRI (IR12 group) and repeated kidney IRI (IR7-12 group), tubular injury still occurred, represented by delayed epithelial cells and an inadequate regeneration process. This result also revealed that there was a repairing process in the recovery phase of kidney IRI. In this phase, cell differentiation continues, epithelial polarity is regained, and organ functions normally return (Hagmann et al. 2011; Harris 1997; Schena 1998). The IR7-12 group represents the model for a maladaptive repair response. Second IRI in the IR7-12 group represented the disruption of the maintenance phase, which leads to a maladaptive repairing process.

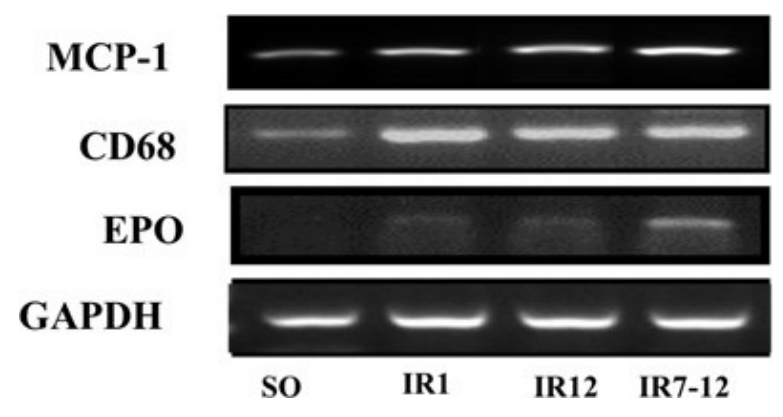

(a)

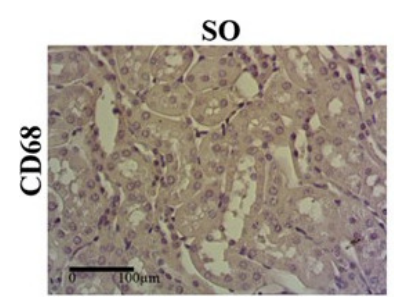

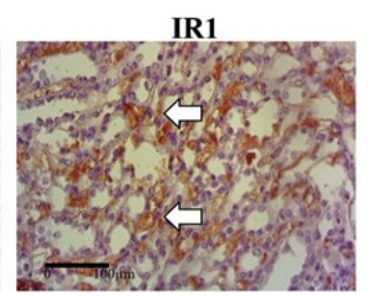

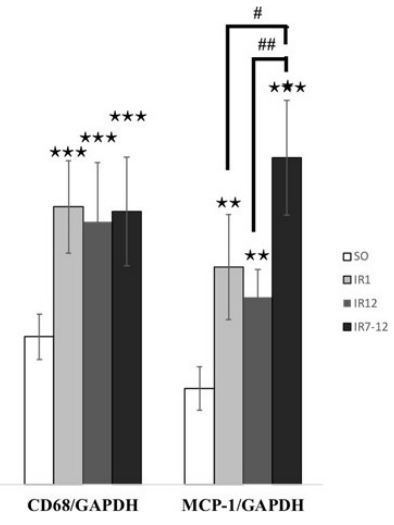

(b)

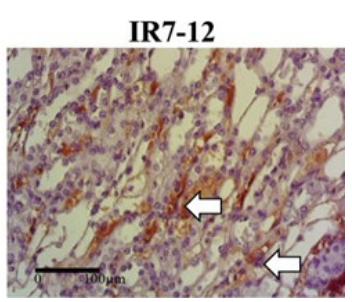

(c)

FIGURE 3 mRNA and protein expression of pro-inflammatory mediators. (a) Gel electrophoresis figures of RT-PCR analyses of MCP-1, EPO, CD68, and GAPDH from kidney tissue. (b) Bar charts of relative quantification for mean EPO/GAPDH, CD68/GAPDH and MCP-1/GAPDH mRNA expressions. Data were analyzed using one-way ANOVA $(p=0.000)$. Asterisks show significances between SO and IRI groups $(* * *, p<$ $0.001 ;{ }^{* *}, p<0.01$ ). Elbow connectors show significances between IRI groups (\#\#, $p<0.001 ; \#, p<0.05$ ). (c) Immunohistochemistry showing CD68 as macrophage marker to demonstrate inflammation (white arrows) in groups IR1, IR12, and IR7-12. Scale bar $=100 \mu \mathrm{m}$. 
Repeated injury in the IR7-12 group also demonstrated the highest number of PDGFR $\beta$-positive cells, followed by the IR12 group. We chose PDGFR $\beta$ as the marker for fibroblasts and pericytes in this study, although there are other markers, such as NG2, that can also be used to examine these two kinds of cells. Both fibroblast expansion and myofibroblast formation not only change fibroblast structure, but its functions, as well (Asada et al. 2011). More hypoxic induces chronic hypoxia, which leads to tubulointerstitial injury (Bonventre and Yang 2011). Maladaptive repair leads to fibroblast activation and tubulointerstitial fibrosis, chronic and excessive profibrotic cytokines production by activated macrophages. IL13 and TGF $\beta-1$ are some of the profibrotic cytokines produced (Bonventre and Yang 2011). Hypoxic inducible factor $1 \alpha$ (HIF-1 $\alpha$ ) may also contribute to the chronic effect of hypoxia that may lead to fibrosis. HIF increases in the acute phase of IRI and provides protective effects in the acute phase of ischemia injury. However, in the renal fibrosis model, deletion of HIF-1 $\alpha$ may prevent epithelial to mesenchymal transition and tubulointerstitial fibrosis (Higgins et al. 2007). Prolonged activation of HIF$1 \alpha$ could also induce the upregulation of profibrotic substances, such as connective tissue growth factor. This upregulation stimulates fibrosis and destruction of renal prenchyme (Haase 2013). Examining fibroblast to myofibroblast formation in this study may shed light on the EPOproducing cells, especially the periods of kidney IRI.

RT-PCR analysis showed an increase of EPO mRNA expression in groups IR1, IR12, and IR7-12. The Pearson correlation test between the number of PDGFR $\beta-$ positive cells and EPO expression showed a significant, strong, positive correlation ( $p=0.000 ; r=+0.795)$. This positive correlation indicated that the higher the number of PDGFR $\beta$-positive cells, the higher the EPO that was produced. Meanwhile, the same test between the number of PDGFR $\beta$-positive cells and hemoglobin concentration showed a significant, strong, negative correlation ( $p=0.000 ; r=-0.822$ ). It can be concluded that the greater the number of PDGFR $\beta$-positive cells, the greater the decrease in hemoglobin levels. This study showed that the correlation between number of PDGFR $\beta$-positive cells with hemoglobin concentration and creatinine level trended in opposite directions. It can be induced by chronic inflammation, which becomes the basis for EPO resistance in CKD models. This condition may need further examination of the proliferation of fibroblasts. Proliferated fibroblasts may transform into myofibroblasts, thus reducing the EPO production from fibroblasts (Asada et al. 2011). In this study, we found that anemia still occurred in spite of the increased mRNA expression of EPO. Further analysis of the phenotype of the fibroblast is necessary for the completion of this study. Double immunostaining to examine the fibroblast to myofibroblast transition or EPO immunostaining may be considered important to continue this research. We consider this matter to be one of the limitations of this study. In CKD cases, anemia can be due to EPO deficiency and resistance, as well as disruption in iron metabolism (Hörl 2013), and it may be related to inflammation.

Inflammation plays a role in the pathogenesis of kidney injuries. Inflammation occurs in both acute and chronic phases of kidney injury, and further contributes to maladaptive responses (Akcay et al. 2009). This process activates proinflammatory cytokines and chemotactic agents, such as TNF- $\alpha$, MCP-1, IL- 8 , IL-6, TGF- $\beta$, regulated on activation, normal $\mathrm{T}$ expressed and secreted (RANTES) protein, and epithelial neutrophil-activating protein 78 (ENA-78), and then induces macrophage infiltration (Basile and Yoder 2014). We elucidated macrophage infiltration through the expression of mRNA and protein CD68 as the macrophage marker. CD68 expression was increased in groups IR1, IR12, and IR712 , and was significantly different from group SO. Immunostaining revealed infiltration of the macrophage in IR groups, which was associated with the upregulation of MCP-1 and CD68 mRNA expression. This result could be due to chronic inflammation in groups IR12 and IR7-12, which leads to chronic and excessive production of profibrotic cytokines. Anemia in CKD is a type of anemia of chronic disease (Weiss and Goodnough 2005). The ACD is characterized by a blunted EPO response, decrease of red blood cells age, and defects in iron metabolism, consisting of decreases in iron absorption and iron retention by macrophages (Weinstein et al. 2002).

Macrophage infiltration leads to macrophage polarization and M1 formation due to inflammatory mediator stimulation. M1 plays a main role in inflammation modulation and inducing tissue damage (Cao et al. 2015). Prolonged inflammation also induces a production on hepcidin in the liver. Hepcidin can degrade transmembrane protein ferroportin (Fpn) in M1. This condition prevents the release of iron into the extracellular environment, and thus iron will undergo sequestration in M1 and the inflammation area. A chronic increase of hepcidin and iron sequestration reduces iron levels in the blood and finally induces anemia (Cairo et al. 2011). Kidney IRI upregulates inflammatory cytokines production, then induces M1 formation and iron sequestration. Proinflammatory cytokines, such as TNF $\alpha$, IL-1 $\beta$, and IL-6, may also upregulate hepcidin (Gammella et al. 2014). Elucidating proteins that contribute to iron metabolism, such as hepcidin or transferrin, may provide a better understanding of the effect of IRI on anemia and EPO production. In the future, it is also necessary to examine the possibility of iron entrapment and deposition in the kidney after IRI.

\section{Conclusions}

In conclusion, chronic effects of single and repeated IRI cause interstitial cells expansion and an elevation in EPO mRNA expression. The decrease in hemoglobin levels could be caused by chronic inflammation that inhibits EPO effectiveness. Therefore, our study revealed that chronic and repeated kidney ischemic/reperfusion injury induced fibroblast expansion and inflammation associated with anemia. 


\section{Acknowledgments}

The authors would like to extend their highest gratitude to Mr. Mulyana for all his kind assistance as the animal facility and laboratory assistant. This research was funded by the University Leading Research Grant (Penelitian Unggulan Perguruan Tinggi) from the Ministry of Research, Technology, and Higher Education of the Republic of Indonesia.

\section{Authors' contributions}

NA, DP and DC designed the study. NA, DP and WA carried out the experimental work in the laboratory, such as the surgical procedures on the mice subjects, immunohistochemistry staining, RNA extraction and PCR analysis. MR and NA analyzed the data. DP and NA wrote the manuscript. All the authors read and approved the final version of the manuscript.

\section{Competing interests}

The authors declare there is no conflict of interest.

\section{References}

Akcay A, Nguyen Q, Edelstein CL. 2009. Mediators of inflammation in acute kidney injury. Mediators Inflamm. 2009:1-12. doi:10.1155/2009/137072.

Arfian N, Emoto N, Vignon-Zellweger N, Nakayama K, Yagi K, Hirata KI. 2012. ET-1 deletion from endothelial cells protects the kidney during the extension phase of ischemia/reperfusion injury. Biochem Biophys Res Commun. 425(2):443-449. doi:10.1016/j. bbrc.2012.07.121.

Asada M N Aand Takase, Nakamura J, Oguchi A, Asada M, Suzuki N, Yamamura K, Nagoshi N, Shibata S, Rao TN, Fehling HJ, Fukatsu A, Minegishi N, Kita T, Kimura T, Okano H, Yamamoto M, Yanagita M. 2011. Dysfunction of fibroblasts of extrarenal origin underlies renal fibrosis and renal anemia in mice. J Clin Invest. 121(10):3981-3990. doi:10.1172/JCI57301.

Basile DP, Yoder MC. 2014. Renal endothelial dysfunction in acute kidney ischemia reperfusion injury. Cardiovasc Hematol Disord: Drug Targets. 14(1):3-14.

Bayati A, Nygren K, Källskog Ö, Wolgast M. 1990. The long-term outcome of post-ischaemic acute renal failure in the rat. II. A histopathological study of the untreated kidney. Acta Physiol Scand. 138(1):35-47. doi:10.1111/j.1748-1716.1990.tb08809.x.

Bergström J, Lindholm B. 2000. What are the causes and consequences of the chronic inflammatory state in chronic dialysis patients? Semin Dial. 13(3):163164. doi:10.1046/j.1525-139x.2000.00044.x.

Bonventre JV, Weinberg JM. 2003. Recent advances in the pathophysiology of ischemic acute renal failure. $\mathrm{J}$ Am Soc Nephrol. 14(8):2199-2210. doi:10.1097/01. ASN.0000079785.13922.F6.
Bonventre JV, Yang L. 2011. Cellular pathophysiology of ischemic acute kidney injury. J Clin Invest. 121(11):4210-4221. doi:10.1172/JCI45161.

Cairo G, Recalcati S, Mantovani A, Locati M. 2011. Iron trafficking and metabolism in macrophages: contribution to the polarized phenotype. Trends Immunol. 32(6):241-247. doi:10.1016/j.it.2011.03.007.

Cao Q, Harris DC, Wang Y. 2015. Macrophages in kidney injury, inflammation, and fibrosis. Am J Physiol. 30(3):183-194. doi:10.1152/physiol.00046.2014.

Donovan A, Lima CA, Pinkus JL, Pinkus GS, Zon LI, Robine S, Andrews NC. 2005. The iron exporter ferroportin/Slc40a1 is essential for iron homeostasis. Cell Metab. 1(3):191-200. doi:10.1016/j.cmet.2005. 01.003.

Gammella E, Buratti P, Cairo G, Recalcati S. 2014. Macrophages: central regulators of iron balance. Metallomics. 6(8):1336-1345. doi:10.1039/C4MT00104D.

Go AS, Chertow GM, Fan D, McCulloch CE, Hsu CY. 2004. Chronic kidney disease and the risks of death, cardiovascular events, and hospitalization. N Engl J Med. 351(13):1296-1305. doi:10.1056/ NEJMoa041031.

Haase VH. 2013. Regulation of erythropoiesis by hypoxiainducible factors. Blood Rev. 27(1):41-53. doi:10. 1016/j.blre.2012.12.003.

Hagmann H, Bossung V, Belaidi AA, Fridman A, Karumanchi SA, Thadhani R, Schermer B, Mallmann P, Schwarz G, Benzing T, Brinkkoetter PT. 2011. Lowmolecular weight heparin increases circulating sFlt-1 levels and enhances urinary elimination. PLoS One. 9(1):1-8. doi:10.1371/journal.pone.0085258.

Harris RC. 1997. Growth factors and cytokines in acute renal failure. Adv Ren Replace Ther. 4(2 Suppl 1):4353.

Higgins DF, Kimura K, Bernhardt WM, Shrimanker N, Akai Y, Hohenstein B, Saito Y, Johnson RS, Kretzler M, Cohen CD, Eckardt KU, Iwano M, Haase VH. 2007. Hypoxia promotes fibrogenesis in vivo via HIF-1 stimulation of epithelial-to-mesenchymal transition. J Clin Invest. 117(12):3810-3820. doi: 10.1172/JCI30487.

Hörl WH. 2013. Anaemia management and mortality risk in chronic kidney disease. Nat Rev Nephrol. 9(5):291301. doi:10.1038/nrneph.2013.21.

Kim J, Jung KJ, Park KM. 2010. Reactive oxygen species differently regulate renal tubular epithelial and interstitial cell proliferation after ischemia and reperfusion injury. Am J Physiol: Renal Physiol. 298(5):11181129. doi:10.1152/ajprenal.00701.2009.

Mason J, Olbricht C, Takabatake T, Thurau K, Pätz S. 1977. The early phase of experimental acute renal failure. Pflügers Arch. 370(2):155-163. doi:10.1007/ BF00581689.

Obara N, Suzuki N, Kim K, Nagasawa T, Imagawa S, Yamamoto M. 2008. Repression via the GATA box is essential for tissue-specific erythropoietin gene ex- 
pression. Blood. 111(10):5223-5232. doi:10.1182/ blood-2007-10-115857.

Park JS, Jo CH, Kim S, Kim GH. 2012. Acute and chronic effects of dietary sodium restriction on renal tubulointerstitial fibrosis in cisplatin-treated rats. Nephrol Dial Transplant. 28(3):592-602. doi:10.1093/ndt/gfs496.

Prodjosudjadi W, Suhardjono A. 2009. End-stage renal disease in Indonesia: treatment development. Ethn Dis. 19(1):33-36.

Schena FP. 1998. Role of growth factors in acute renal failure. Kidney Intl Suppl. 66:11-15.

Stenvinkel P. 2001. The role of inflammation in the anaemia of end-stage renal disease. Nephrol Dial Transplant. 16(suppl 7):36-40. doi:10.1093/ndt/16. suppl_7.36.

Strutz F, Zeisberg M. 2006. Renal fibroblasts and myofi- broblasts in chronic kidney disease. J Am Soc Nephrol. 17(11):2992-2998. doi:10.1681/ASN.2006050420.

Sutton TA, Fisher CJ, Molitoris BA. 2002. Microvascular endothelial injury and dysfunction during ischemic acute renal failure. Kidney Int. 62(5):1539-1549. doi: 10.1046/j.1523-1755.2002.00631.x.

Weinstein DA, Roy CN, Fleming MD, Loda MF, Wolfsdorf JI, Andrews NC. 2002. Inappropriate expression of hepcidin is associated with iron refractory anemia: implications for the anemia of chronic disease. Blood. 100(10):3776-3781. doi:10.1182/ blood-2002-04-1260.

Weiss G, Goodnough LT. 2005. Anemia of chronic disease. N Engl J Med. 352(10):1011-1023. doi:10.1056/ NEJMra041809. 